\title{
Inhibition of NAMPT decreases cell growth and enhances susceptibility to oxidative stress
}

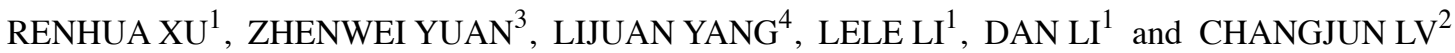 \\ ${ }^{1}$ School of Nursing, Binzhou Medical University, Yantai, Shandong 264003; ${ }^{2}$ Department of Respiratory Medicine, \\ Affiliated Hospital of Binzhou Medical University, Binzhou, Shandong 256603; ${ }^{3}$ Library, Binzhou Medical University, \\ Yantai, Shandong 264003; ${ }^{4}$ Cancer Research Institute, Affiliated Hospital of Binzhou Medical University, \\ Binzhou, Shandong 256603, P.R. China
}

Received December 27, 2016; Accepted June 28, 2017

DOI: $10.3892 /$ or.2017.5793

\begin{abstract}
Nicotinamide adenine dinucleotide (NAD) is an essential molecule for living organisms and plays a vital role in aging and age-associated diseases. In eukaryotic cells, cellular NAD is mainly generated by the scavenge pathway in which nicotinamide phosphoribosyltransferase (NAMPT) catalyzes the formation of nicotinamide mononucleotide. Inhibition of NAMPT is a therapeutic strategy for cancer treatment. To explore the effects of NAMPT inhibition on cellular processes, cells were treated with $10 \mathrm{nM}$ FK866, an NAMPT inhibitor, resulting in a decrease in the cellular NAD level, a lower growth rate, and enhanced susceptivity to oxidative stress as compared to the untreated cells. Quantitative proteomics revealed that 325 proteins were downregulated in the FK866-treated cells, and were involved in diverse cellular processes including nucleobase-containing compound metabolic process, protein metabolic process, antioxidant and DNA repair processes. Downregulation of 4 selected proteins was confirmed by western blotting and quantitative PCR. Downregulation of antioxidant proteins GRX1 and catalase, and DNA-repair proteins PCNA and PARP1 contributed to the enhanced susceptibility of FK866-treated cells to oxidative stress. FK866 treatment also caused mitochondrial dysfunction through downregulation of mitochondrial ribosomal proteins. Taken together, these results demonstrate that FK866 treatment efficiently decreases the cellular NAD level and induces autonomous changes in proteostasis, leading to cell growth inhibition and increased susceptibility to oxidative stress.
\end{abstract}

Correspondence to: Professor Renhua Xu, School of Nursing, Binzhou Medical University, Yantai, Shandong 264003, P.R. China E-mail:xrhazz@163.com

Professor Changjun Lv, Department of Respiratory Medicine, Affiliated Hospital of Binzhou Medical University, Binzhou, Shandong 256603, P.R. China

E-mail: lucky_lcj@sina.com

Key words: FK866, proteomics, NAD, oxidative stress, NAMPT

\section{Introduction}

NAD is an essential component in life, which plays a vital role in major biological processes (1-3). In cells, the NAD pool is composed of NAD, reduced nicotinamide adenine dinucleotide (NADH), nicotinamide adenine dinucleotide phosphate (NADP) and reduced nicotinamide adenine dinucleotide phosphate (NADPH). NAD and NADP are coenzymes for dehydrogenase (DH)-catalyzed reactions involved in biodegradation and biosynthesis. The NAD/NADH ratio also regulates mitochondrial functions (4-6). NADPH plays an important role in the cell defense system. NAD homeostasis is maintained by NAD synthesis, NAD catabolism, and non-redox NAD-dependent enzymatic reactions. Tryptophan is the precursor for de novo NAD synthesis.

In non-redox NAD-dependent reactions, NAD functions as a substrate for ADP-ribosyltransferases such as poly(ADP-ribose) polymerases (PARPs), mono(ADP-ribosyl)-transferases (ARTs), NAD(+)-dependent deacetylases (sirtuins), tRNA 2'-phosphotransferases, and ADP-ribosyl cyclases. Sirtuins utilize NAD to deacetylate acetyl-lysine residues in proteins (7-10). Sirtuin-catalyzed NAD-dependent deacetylations of nuclear and mitochondrial proteins are the basis for the broad range of cellular functions (11-14). As the substrate for ADP-ribosylation of nuclear proteins, NAD is essential for stress responses $(15,16)$. Recent studies have revealed that NAD levels decline with age in different organisms (17-20). On the other hand, repletion of NAD precursors increases the cellular NAD levels against age-associated diseases and prolongs lifespan extension (21-23). A recent study demonstrated that CD38 levels are increased in aged mice, causing age-related NAD decline and mitochondrial dysfunction (24).

Furthermore, NAD is essential to tumor survival and progression by regulating multiple cellular processes, including metabolism, stress responses, and DNA integrity. Targeting NAD metabolism has evolved to be a new concept in cancer therapy $(25,26)$. The cellular NAD levels are maintained by the NAD scavenge pathway in which the formation of nicotinamide mononucleotide is the rate-limiting step catalyzed by nicotinamide phosphoribosyltransferase (NAMPT). It has been amply documented that NAMPT expression is 
highly expressed in tumors, making it a therapeutic target in cancer treatment $(26,27)$. Over the last two decades, extensive studies have identified that FK866 efficiently inhibits NAMPT activity to decrease cellular NAD levels $(28,29)$. Studies show that FK866-induced cell growth inhibition is regulated by $\mathrm{p} 53$, mTOR and autophagy pathways (30-34). However, few studies have systematically examined the effects of NAD decrease on proteostasis, cell proliferation and stress responses. Understanding the global impacts of the NAD levels on cellular processes is important for treating cancer and age-associated diseases. In the present study, we treated cells with FK866 and conducted a comprehensive analysis to decipher the effects of the decrease in NAD on proteostasis, cell growth, and cellular responses to oxidative stress.

\section{Materials and methods}

Chemicals and reagents. Phosphate-buffered saline (PBS), penicillin/streptomycin, Dulbecco's modified Eagle's medium (DMEM), normal and dialyzed fetal bovine serum were all purchased from Wisent (Montreal, QC, Canada). SILAC labeling reagents including DMEM medium, isotope encoded $\mathrm{L}_{-13}{ }^{13} \mathrm{C}_{6}$ lysine $\cdot \mathrm{HCl}$, standard $\mathrm{L}-$ lysine $\cdot \mathrm{HCl}$, and standard L-arginine. $\mathrm{HCl}$ were purchased from Thermo Fisher (Waltham, MA, USA). Sequencing grade trypsin was purchased from Promega (Fitchburg, WI, USA). Iodoacetamide (IAA) and polybrene were purchased from Sigma (St. Louis, MO, USA). Anti-PARP1 antibody, anti-PCNA antibody and anti- $\beta$-actin antibody were purchased from Abmart (Shanghai, China). Anti-p53 antibody, anti-mouse secondary antibody and anti-rabbit secondary antibody were purchased from Cell Signaling Technology (Boston, MA, USA). The Cell Counting Kit-8 (CCK-8) was purchased from Dojindo (Kumamoto, Japan). Hydrogen peroxide was purchased from Aladdin (Shanghai, China). BCA protein assay kit was purchased from Solarbio (Beijing, China). The Total RNA isolation and reverse transcription kit were purchased from Tiangen (Beijing, China).

Cell culture and SILAC labeling. Human 293T and A549 cell lines were purchased from the Cell Bank of the Chinese Academy of Sciences (Shanghai, China). The cells were grown in DMEM medium that was supplemented with $10 \%$ fetal bovine serum (FBS) and $1 \%$ penicillin/streptomycin at $37^{\circ} \mathrm{C}$ in a humidified incubator with $5 \% \mathrm{CO}_{2}$. For SILAC labeling, 293T cells were washed twice with PBS prior to being cultured with the SILAC culture medium, which was prepared by mixing SILAC DMEM medium with $10 \%$ D-FBS, $1 \%$ penicillin/ streptomycin, $146 \mathrm{mg} / 1$ isotope labeling $\mathrm{L}-{ }^{13} \mathrm{C}_{6}$ lysine $\cdot \mathrm{HCl}$ and $84 \mathrm{mg} / \mathrm{l}$ standard $\mathrm{L}$-arginine $\cdot \mathrm{HCl}$. The cells were grown for 8 to 10 passages in SILAC medium and the full incorporation of isotope-encoded amino acid into proteins was tested. Cells were cultured for at least $12 \mathrm{~h}$ prior to FK866 treatment; cells were treated with FK866 dissolved in DMSO for 12 or $24 \mathrm{~h}$. After treatments, the cells were then washed twice with icecold PBS and lysed with RIPA lysis buffer which consisted of $25 \mathrm{mmol} / 1 \mathrm{Tris}-\mathrm{HCl} \mathrm{pH} 7.6,150 \mathrm{mmol} / \mathrm{l} \mathrm{NaCl}, 0.1 \%$ SDS, $1 \%$ NP-40, $1 \%$ sodium deoxycholate, $1 \mathrm{mmol} / 1 \mathrm{PMSF}$, and Roche Complete Protease Inhibitor Cocktail for $30 \mathrm{~min}$ on ice. Cell lysates were further clarified by centrifugation at $14,000 \times \mathrm{g}$ for $20 \mathrm{~min}$ at $4^{\circ} \mathrm{C}$. The protein concentration in each sample was determined by using a BCA protein assay kit.

Analysis of NAD and NADH content. The NAD and NADH contents were determined using a NAD/NADH quantification kit according to the manufacturer's instructions (http://www. sigmaaldrich.com/content/dam/sigma-aldrich/docs/Sigma/ Bulletin/1/mak037bul.pdf). Briefly, after cells were washed three times with cold PBS, cell metabolites were extracted using the NADH/NAD extraction buffer for 3 freeze/thawing cycles. After centrifugation, the supernatant was collected. For total NAD (NAD + NADH) detection, the supernatant was mixed with the NAD cycling enzyme to convert NAD to NADH. For NADH detection, the supernatant was heated to $60^{\circ} \mathrm{C}$. Then, analytes and NADH standards reacted with a $\mathrm{NADH}$ developer reagent. The products were measured at the absorbance wavelength $450 \mathrm{~nm}$ (A450) to quantify NAD and NADH concentrations.

NAD and NADH concentrations were also measured by LC-MS analysis. Briefly, the cells were washed with ice-cold PBS and the metabolites were extracted using $80 \%$ methanol $\left(-80^{\circ} \mathrm{C}\right)$. The metabolites were dried using a speedvac and re-dissolved in $80 \%$ methanol and used for LC-MS. The metabolites were separated by a Acquity UPLC BEH amide column (2.1x100 mm, 1.7- $\mu \mathrm{m}$; Waters, Milford, MA, USA) that was interfaced with the Q-Exactive Mass spectrometer. Metabolites were characterized based on the retention time and the accurate mass measurement with $<5$ ppm mass accuracy. Trace Finder software was employed to identify the peaks and extract the quantitative information.

Cell proliferation measurement with $C C K-8$ kit. The cells were seeded in 96-well plates at 2,000 cells/well. The cell proliferation rate was measured with the CCK- 8 kit according to the manufacturer's protocol (Dojindo Laboratories). Briefly, CCK-8 reagents were added into each well after cells grew for $0,8,16,24,32,40,48,72$, and $96 \mathrm{~h}$, respectively. The absorbance at $450 \mathrm{~nm}$ was measured $3 \mathrm{~h}$ after CCK-8 addition.

Susceptibility of FK866-treated and untreated cells to hydrogen peroxide and cisplatin. Effects of hydrogen peroxide and cisplatin on cell growth in the FK866-treated and untreated cells were analyzed with the CCK- 8 kit. Briefly, cells were seeded into 96-well cell culture microplates and incubated with $10 \mathrm{nM}$ FK866 for $6 \mathrm{~h}$ prior to $\mathrm{H}_{2} \mathrm{O}_{2}$ or cisplatin treatment. Then, untreated and FK866-treated cells were treated with $\mathrm{H}_{2} \mathrm{O}_{2}(200,400$, and $600 \mu \mathrm{M})$ or cisplatin at different concentrations $(10,25$ and $50 \mu \mathrm{M})$ in triplicates for $12 \mathrm{~h}$. Afterwards, CCK- 8 reagents were added to the treated cells and incubated for $3 \mathrm{~h}$ at $37^{\circ} \mathrm{C}$. The optical density (OD) was measured at $450 \mathrm{~nm}$ with a microplate reader (Bio-Rad, Hercules, CA, USA). Cell viability is represented as the percentage of viable cells compared to that of the untreated cells. The experiment was repeated 3 times.

Identification of differentially expressed proteins by proteomic analysis. Proteomic analysis was conducted as previously described (35). Briefly, equal amounts of proteins from the FK866-treated and the untreated cells were mixed together and separated by $1 D$ SDS-PAGE. The gel bands were excised, 
A

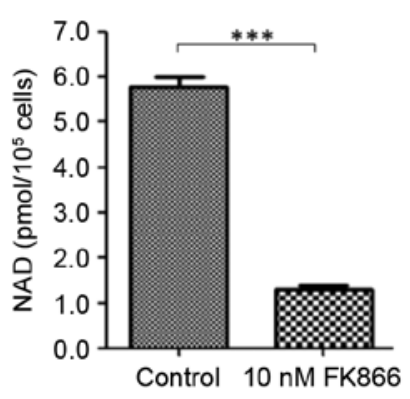

B

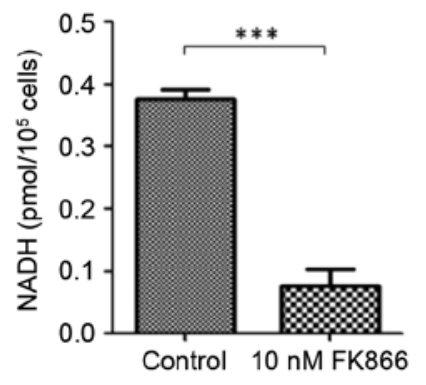

C

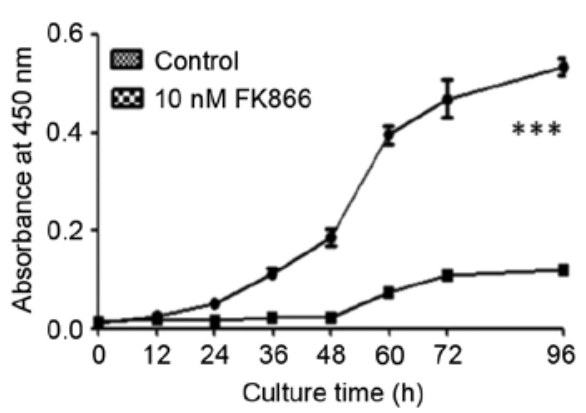

Figure 1. FK866 treatment decreases the cellular NAD levels and inhibits cell proliferation. (A) Graphical representation of mean values and the standard deviation of the cellular NAD levels in the untreated and FK866-treated 293T cells. (B) Cellular NADH levels in the untreated and FK866-treated cells. (C) Cell proliferation rate as determined by the CCK- 8 assay. ${ }^{* * *} \mathrm{p}<0.01 ; \mathrm{n}=3$.

reduced with $25 \mathrm{mM}$ DTT and alkylated with $55 \mathrm{mM}$ IAA, followed by in-gel digestion with sequencing grade trypsin in $40 \mathrm{mM}$ ammonium bicarbonate at $37^{\circ} \mathrm{C}$ overnight. The peptides were extracted twice and analyzed.

The digestion product was analyzed by LC-MS/MS, in which peptides were separated by a 65 -min gradient elution at a flow rate $0.250 \mu \mathrm{l} / \mathrm{min}$ with an Dionex Ultimate integrated nano-HPLC system which was directly interfaced with a Thermo Orbitrap Q-Exactive mass spectrometer. The Q-Exactive mass spectrometer was operated with the data-dependent acquisition method. A full-scan followed by 20 data-dependent MS/MS scans were acquired with normalized collision energy at 33\%. Proteomic analysis was conducted in biological triplicates. The SEQUEST searching algorithm in the Proteome Discoverer software (version 1.4.1.14) was used to generate the peak lists from LC-MS/MS analysis, which were searched against the Uniprot Human database (release date of November 20, 2015; 20,193 entries). The search criteria were the following: full tryptic specificity was required; one missed cleavage was allowed; carbamidomethylation (C) was set as the fixed modifications; the oxidation (M) was set as the variable modification; precursor ion mass tolerances were set at 10 ppm for all MS acquired in an orbitrap mass analyzer; and the fragment ion mass tolerance was set at 0.02 Da for all MS2 spectra acquired. The peptide false discovery rate was evaluated using the Percolator provided by Proteome Discoverer software. When the q-value was smaller than $1 \%$, the match was considered to be correct. False discovery was evaluated based on peptide spectrum match when searched against the decoy database. Peptides only assigned to a given protein group were considered as unique. Protein quantitation was also conducted with Proteome Discoverer Algorithm (version 1.4). Briefly, the ratio in relative protein expression for each lysine-containing peptide was calculated using the peak area of Lys6 divided by the peak area of Lys0. The protein ratio was then averaged with all peptide ratios for that protein. Quantitative precision was expressed as protein ratio variability.

Western blot analysis. FK866-treated and the untreated cells were harvested and lysed on ice with RIPA lysis buffer. The supernatants were then collected and the protein concentrations were measured with the BCA protein assay kit. Proteins were separated on 1D SDS-PAGE gel and transferred onto a
PVDF membrane with electroblotting. The PVDF membrane was blocked with $5 \%$ nonfat milk for $1 \mathrm{~h}$ at room temperature and incubated with primary antibody overnight at $4^{\circ} \mathrm{C}$, washed three times with TBST buffer, and then incubated with anti-mouse or anti-rabbit secondary antibody labeled with HRP. The PVDF membrane was then washed three times with TBST buffer and developed with ECL reagents (Engreen, China). $\beta$-actin was used as an internal control and detected with the anti- $\beta$-actin antibody.

Quantitative real-time PCR ( $P P C R)$. Untreated and FK866-treated cells were cultured. Total RNA was extracted with the Total RNA isolation kit. cDNA was synthesized from $3 \mu \mathrm{g}$ total RNA using the Reverse Transcription kit. All qPCR experiments were performed with the Roche LightCycler $^{\circledR}$ 480II detection system with SYBR-Green incorporation according to the manufacturer's instructions and $\beta$-actin was used as an internal control. The primers were designed and acquired from the Primer Bank (http://pga.mgh. harvard.edu/primerbank/).

Statistical method. Statistical analysis was performed by using GraphPad Prism 5.0 software. Significant differences were determined by the Student's t-test. p-values of $<0.05$ were considered to be significant.

\section{Results}

FK866 treatment decreases cellular NAD levels and growth rate but increases cell susceptibility to oxidative stress. To examine the effects of FK866 on cellular NAD levels, 293T and A549 cells were treated with FK866 at different concentrations for different periods of time. After FK866 treatment, cells were lysed and cellular metabolites were extracted followed by enzymatic assay or LC-MS analysis. The cellular NAD and NADH levels were determined, showing that the NAD level in the FK866-treated cells was $~ 5$ times lower than that in the 293T cells. Similarly, the level of NADH was found to be 4 times lower in the FK866-treated cells than that in the 293T cells (Fig. 1A and B). Similar results were obtained by LC-MS analysis using the extracted ion currents from mass spectrometric analysis (data not shown). Cell proliferation rates for 293T and FK866-treated cells were determined using 

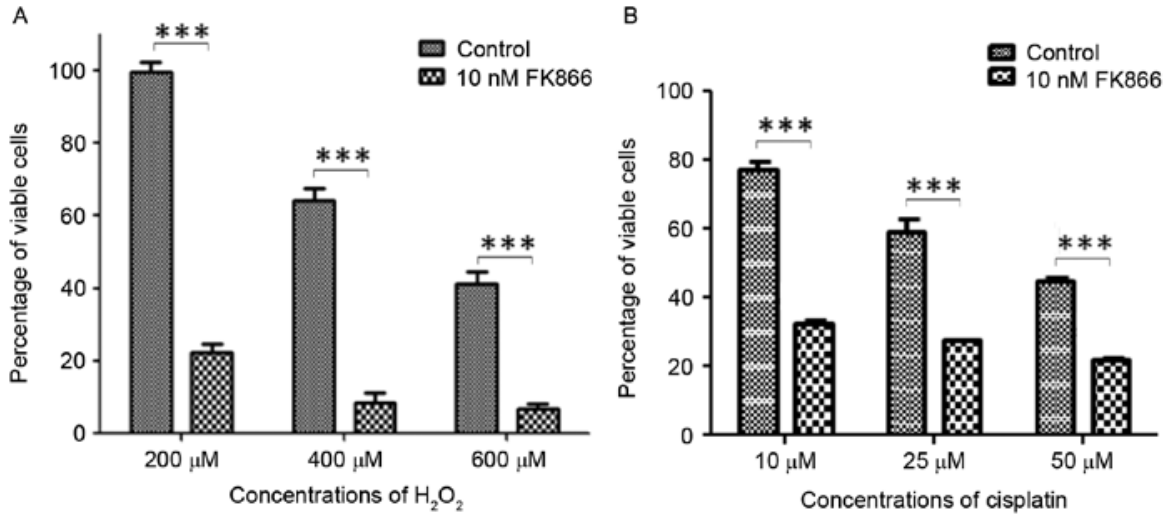

Figure 2. FK866 treatment increases the cell susceptibility to oxidative stress in $293 \mathrm{~T}$ cells. (A) Percentages of viable cells treated with hydrogen peroxide at different concentrations for $12 \mathrm{~h}$ as determined by the CCK-8 assay. (B) Percentages of viable cells treated with cisplatin at different concentrations for $12 \mathrm{~h}$ as determined by the CCK-8 assay. ${ }^{* * *} \mathrm{p}<0.01 ; \mathrm{n}=3$.
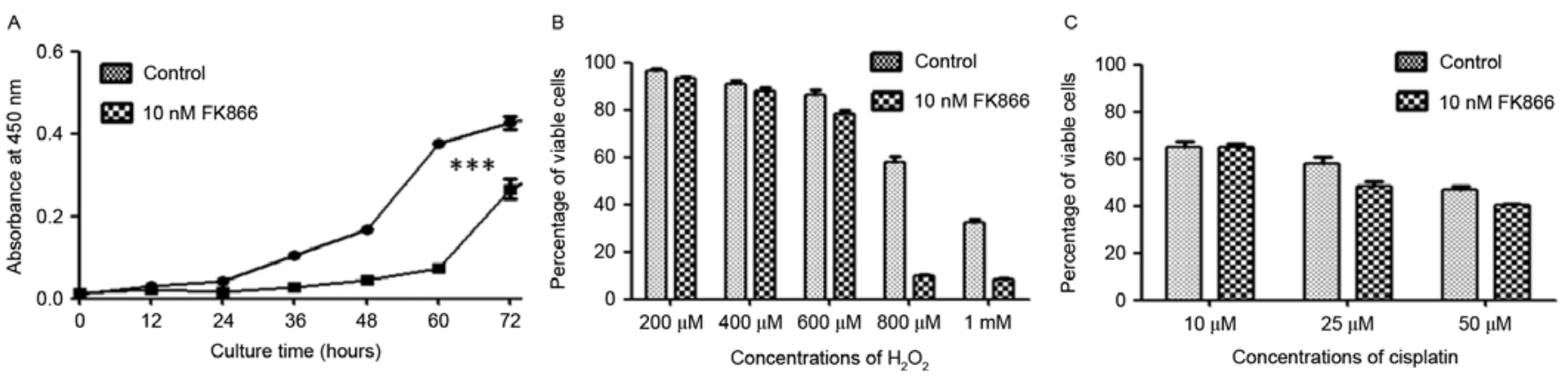

Figure 3. FK866 treatment inhibits cell proliferation and increases the cell susceptibility to oxidative stress in A549 cells. (A) Cell proliferation rate as determined by the CCK-8 assay. (B) Percentages of viable A549 cells treated with hydrogen peroxide at different concentrations for $12 \mathrm{~h}$ as determined by the CCK-8 assay. (C) Percentages of viable cells treated with cisplatin at different concentrations for $12 \mathrm{~h}$ as determined by CCK-8 assay. ${ }^{* * *} \mathrm{p}<0.01 ; \mathrm{n}=3$.

the CCK-8 assay (Fig. 1C) which is a colorimetric assay for analysis of viable cells. The FK866-treated cells grew more slowly than $293 \mathrm{~T}$ cells. At $96 \mathrm{~h}$, the number of $293 \mathrm{~T}$ cells was $\sim 5$ times more than that of the FK866-treated cells.

To determine the susceptibility of $293 \mathrm{~T}$ and FK866-treated cells to hydrogen peroxide and cisplatin, cells were pretreated with DMSO or FK866 followed by incubation with different concentrations of hydrogen peroxide or cisplatin for $12 \mathrm{~h}$. The cell viability was determined by the CCK- 8 assay. The effects of hydrogen peroxide or cisplatin on the cell viability are represented as the percentage of viable cells after a $12 \mathrm{~h}$ treatment (Fig. 2). When cells were treated with $200 \mu \mathrm{M}$ $\mathrm{H}_{2} \mathrm{O}_{2}$ for $12 \mathrm{~h}$, the percentages of viable cells were 20 and 95\% for the FK866-treated and untreated cells, respectively. The percentage of viable cells decreased to $10 \%$ when FK866-treated cells were treated with $400 \mu \mathrm{M} \mathrm{H}_{2} \mathrm{O}_{2}$ for $12 \mathrm{~h}$, indicating that FK866 treatment made 293T cells extremely sensitive to $\mathrm{H}_{2} \mathrm{O}_{2}$ treatment. When cells were treated with $50 \mu \mathrm{M}$ cisplatin for $12 \mathrm{~h}$, the percentages of viable cells were 20 and $50 \%$ for FK866-treated and the untreated cells, respectively. Similar results were also obtained for FK866-treated A549 cells, in which FK866 treatment inhibited the growth of A549 cells while increased cell susceptibility to oxidative stress (Fig. 3).

Identification of differentially expressed proteins between $293 T$ and FK866-treated cells. We then carried out proteomic analysis to identify differentially expressed proteins between 293T and FK866-treated cells. Equal amounts of proteins from 293 T and FK866-treated cells were pooled and separated by 1D SDS-PAGE. SILAC-based quantitation was used to determine the differentially expressed proteins. The experiments were repeated three times and $\sim 4,200$ proteins were identified in each experiment. The false-positive rate was estimated to be $<1 \%$. Based on SILAC ratios ( $>1.5$ or $<0.67), 384$ proteins were found to be differentially expressed between $293 \mathrm{~T}$ and FK866-treated cells, in which 325 proteins were downregulated and 59 were upregulated (data not shown). In order to understand the biological relevance of the identified proteins, Gene Ontology (GO) was employed to cluster the downregulated proteins according to their biological processes which are summarized via a pie plot using the PANTHER bioinformatics platform (http://www.pantherdb.org/) (Fig. 4). Three hundred and twenty-five proteins were classified into several significant groups of biological processes according to their molecular functions including primary metabolism, amino acid metabolism, DNA repair, and cell cycle regulation.

Verification of differentially expressed proteins by western blotting and qPCR. Among the differentially expressed proteins, protein expression levels of PARP1, p53 and GRX1 were lower in the FK866-treated cells than those in the untreated cells, whereas angiomotin expression was higher. Western blot analysis confirmed that the FK866 treatment 


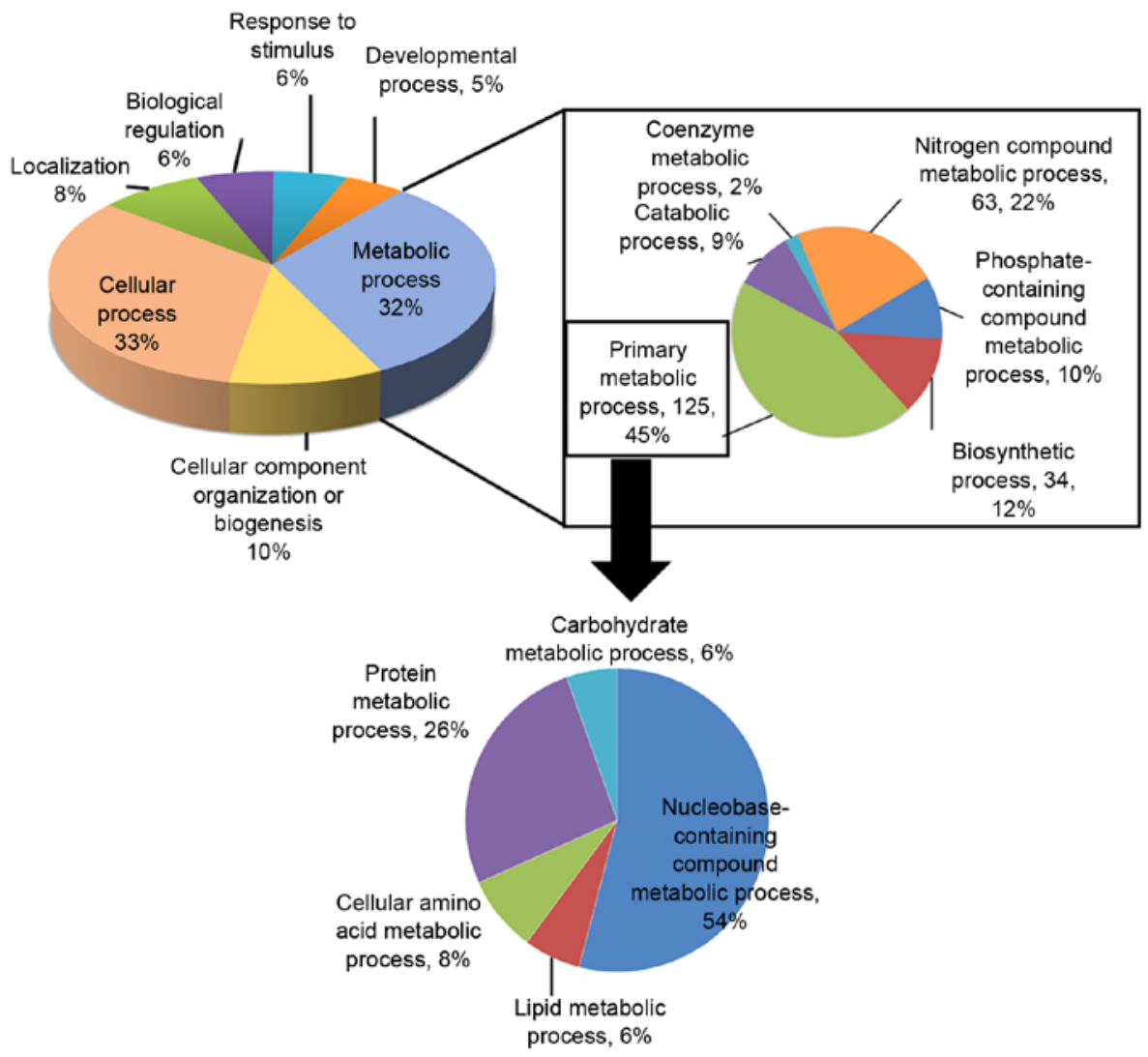

Figure 4. Functional classification of differentially expressed proteins between the untreated and FK866-treated cells with PANTHER (http://www.pantherdb.org).

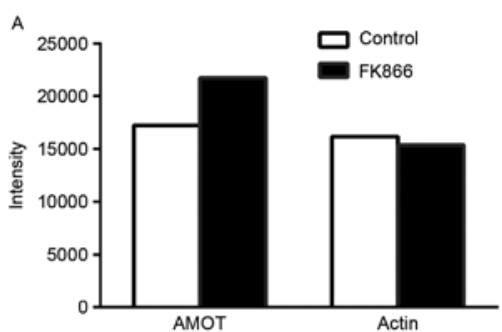

C
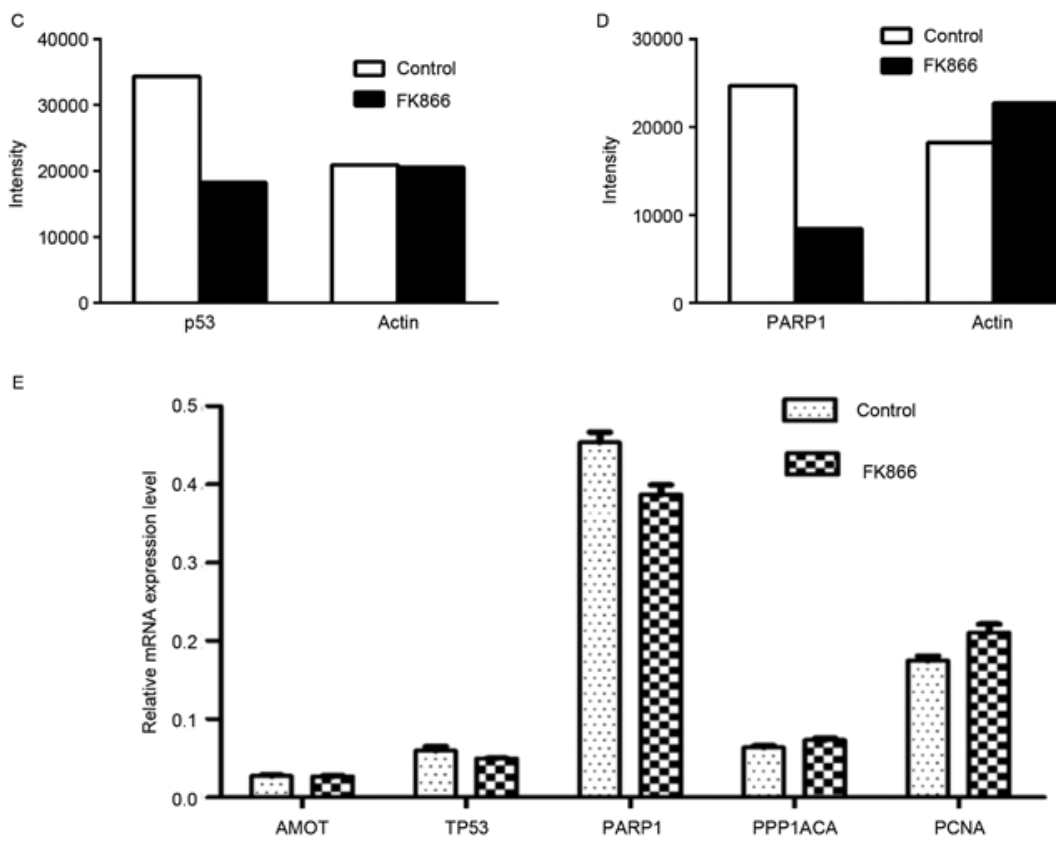

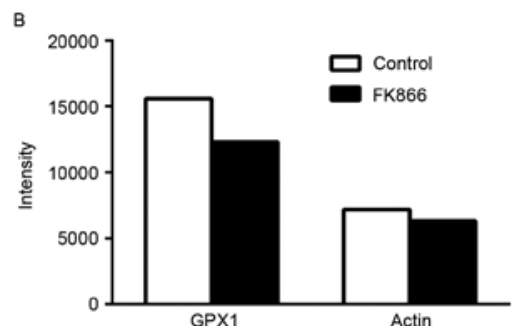

D

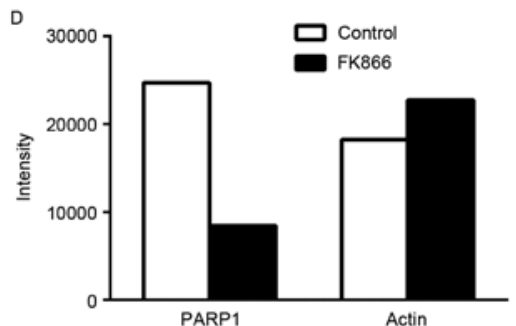

Figure 5. Confirmation of differentially expressed proteins by western blotting and qPCR. (A-D) Band intensities from western blot images of the selected proteins from the untreated and FK866-treated cells. (E) qPCR analysis of mRNA expression of selected genes from the untreated and FK866-treated cells. 

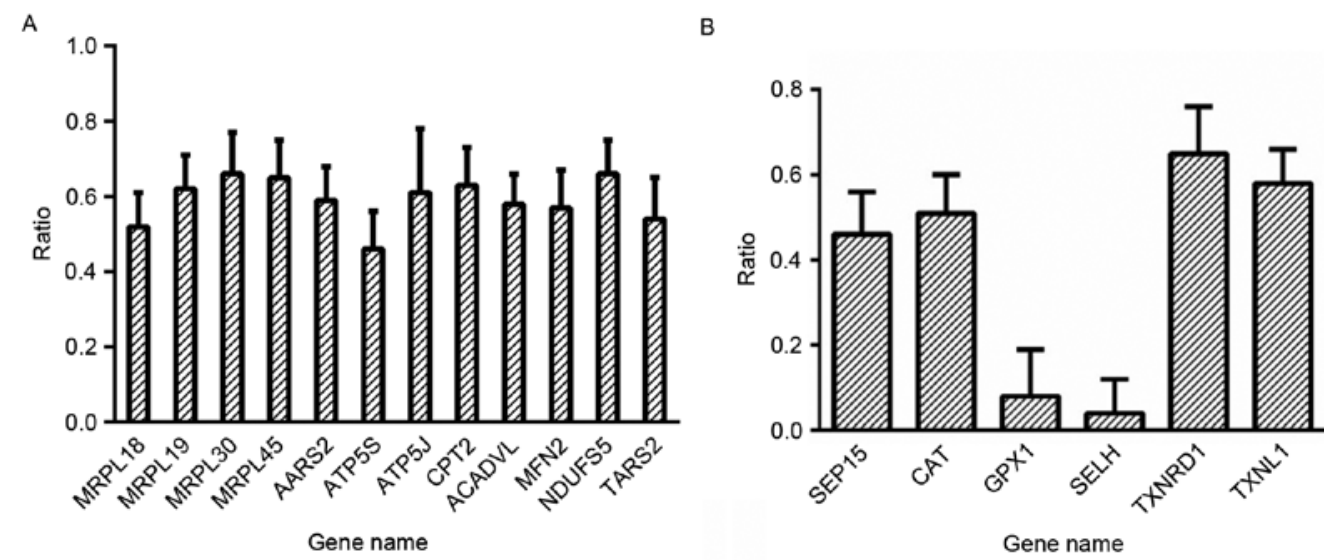

Figure 6. (A) Graphical representation of SILAC ratios for the selected mitochondrial proteins in FK866-treated cells compared to the control cells. (B) Graphical representation of SILAC ratios for the selected antioxidant proteins in FK866-treated cells compared to the control cells.

induced downregulation of PARP1, p53, and GRX1, and angiomotin upregulation (data not shown). Band intensities in western blot images were quantified using Image Lab 4.0.1 software (Fig. 5A-D). The changes in the band intensities from western blotting were comparable to the SILAC ratios. Moreover, qPCR analysis was employed to quantify changes in mRNA levels of the selected genes. Results showed that the mRNA expression levels of p53 and PARP1 genes were lower in the FK866-treated cells than those in the untreated cells (Fig. 5E). However, downregulation of mRNA expressions of p53 and PARP1 genes was less significant than the corresponding changes in protein expression.

\section{Discussion}

NAD plays an important role in all aspects of biological processes and its cellular levels are regulated by both NAD synthesis and consumption. To characterize the effects of the NAD decrease on cell proliferation, cell responses to oxidative stress, and protein homeostasis, we treated cells with FK866, an NAMPT inhibitor. FK866 treatment led to an $80 \%$ decrease in the cellular NAD level. Similarly, the level of NADH was also significantly decreased in the FK866treated cells as compared to the untreated cells (Fig. 1B). By measuring the proliferation rates of the untreated and FK866treated cells (Fig. 1C), we observed that FK866-treated cells grew slower than that observed in the untreated cells, indicating that the lower cellular NAD and NADH levels in the FK866-treated cells slowed down cell proliferation. We measured the cell survival rates after treating the untreated and FK866-treated cells with hydrogen peroxide and cisplatin, and found that FK866-treated cells were extremely sensitive to oxidative stress induced by hydrogen peroxide and cisplatin. This indicates that the cellular NAD levels directly affect the responses to oxidative stress.

To identify factors leading to cell growth inhibition and enhanced cell susceptibility to oxidative stress in the FK866-treated cells, we used the SILAC method to quantify proteins differentially expressed between the untreated and FK866-treated cells. We identified approximately 4,000 proteins in three repeated experiments. Among them, 384 proteins were differentially expressed between the untreated and FK866-treated cells, and these were found to participate in a variety of cellular processes, including RNA process, cell metabolism, stress responses, and cell cycle control and DNA repair. Based on GO analysis, 47 downregulated proteins were associated with carbohydrate metabolisms, nucleobase-containing compound metabolic process, and protein metabolism, suggesting that the cellular NAD level adversely affected cellular metabolic process. Quantitative proteomics also showed that proteins associated with mitochondrial mRNA translation were downregulated in the FK866-treated cells, including mitochondrial ribosome subunits, alanine-tRNA ligase, threonine-tRNA ligase and ATP synthase subunits (Fig. 6A), indicating that mitochondrial protein translation was impaired in the FK866-treated cells. These results suggest that downregulation of proteins associated with primary metabolic process and mitochondrial protein synthesis contributes to the FK866-mediated growth inhibition.

Quantitative proteomics also showed that six antioxidant proteins $15 \mathrm{kDa}$ selenoprotein (Sep15), thioredoxin reductase 1 (TXNRD1), selenoprotein H (SELH), catalase (CAT), glutathione peroxidase 1 (GPX1) and thioredoxin-like protein 1 (TXNL1) were downregulated in the FK866treated cells (Fig. 6B). These proteins are parts of the cellular defense system against oxidative stress: GPX1 catalyzes the reduction of $\mathrm{H}_{2} \mathrm{O}_{2}$ and other hydroperoxides and protects cells from reactive oxygen metabolites; and SELH is a novel nucleolar oxidoreductase that protects cells from oxidative damage (36-38). Downregulation of antioxidant proteins in the FK866-treated cells confirmed that the treated cells were more sensitive to oxidative stress. It is worth noting that FK866mediated PARP1 downregulation was identified by both proteomics and western blotting. PARP1 plays an important role in regulating the cellular NAD level. PARP1 interacts with nuclear NAD synthase to increase the local NAD concentration that regulates PARP1-dependent gene expression, and PARP1 knockdown can increase the cellular NAD level $(39,40)$. Our results revealed that a decrease in NAD induced autonomous PARP1 downregulation. Furthermore, downregulation of PARP1 and other DNA repair proteins, including RAD51, ALKBH1, and HMGB2 evidently led to the increased susceptibility to oxidative stress in the FK866-treated cells. 
In conclusion, the present study investigated the effects of the cellular NAD content on cellular responses by quantitative proteomics. The cellular NAD level was decreased by $80 \%$ in the FK866-treated cells, which significantly altered the protein homeostasis. Our data indicated that the low cellular NAD level impairs the primary metabolic process and mitochondrial protein translational machinery, leading to the growth inhibition in FK866-treated cells. Downregulation of antioxidant proteins and DNA-repairing proteins contributed to the enhanced susceptibility of the FK866-treated cells to oxidative stress.

\section{Acknowledgements}

We thank the Proteomics Facility at Tsinghua University for providing proteomic analysis. This study was supported by Projects of the Natural Science Foundation of Shandong Province (ZR2016CL08, to RH.X.), Medical and Health Technology Development Program of Shandong Province (2016WS0013, to RH.X.), and the National Natural Science Foundation of China (81670064, to CJ.L.).

\section{References}

1. Yang Y and Sauve AA: NAD(+) metabolism: Bioenergetics, signaling and manipulation for therapy. Biochim Biophys Acta 1864: 1787-1800, 2016.

2. Verdin E: $\mathrm{NAD}^{+}$in aging, metabolism, and neurodegeneration. Science 350: 1208-1213, 2015 .

3. Imai S and Guarente L: NAD ${ }^{+}$and sirtuins in aging and disease. Trends Cell Biol 24: 464-471, 2014.

4. Viña J, Saez GT, Gambini J, Gomez-Cabrera MC and Borrás C: Role of $\mathrm{NAD}(+) / \mathrm{NADH}$ redox ratio in cell metabolism: A tribute to Helmut Sies and Theodor Bücher and Hans A. Krebs. Arch Biochem Biophys 595: 176-180, 2016.

5. Ying W: $\mathrm{NAD}^{+} / \mathrm{NADH}$ and $\mathrm{NADP}^{+} / \mathrm{NADPH}$ in cellular functions and cell death: Regulation and biological consequences. Antioxid Redox Signal 10: 179-206, 2008.

6. Xia W, Wang Z, Wang Q, Han J, Zhao C, Hong Y, Zeng L, Tang L and Ying W: Roles of NAD(+)/NADH and NADP(+)/NADPH in cell death. Curr Pharm Des 15: 12-19, 2009.

7. Blander G and Guarente L: The Sir2 family of protein deacetylases. Annu Rev Biochem 73: 417-435, 2004.

8. Moazed D: Enzymatic activities of Sir2 and chromatin silencing. Curr Opin Cell Biol 13: 232-238, 2001.

9. Denu JM: The Sir 2 family of protein deacetylases. Curr Opin Chem Biol 9: 431-440, 2005.

10. Guarente L: Sirtuins and calorie restriction. Nat Rev Mol Cell Biol 13: 207, 2012.

11. Verdin E, Hirschey MD, Finley LW and Haigis MC: Sirtuin regulation of mitochondria: Energy production, apoptosis, and signaling. Trends Biochem Sci 35: 669-675, 2010.

12. Brooks CL and Gu W: How does SIRT1 affect metabolism, senescence and cancer? Nat Rev Cancer 9: 123-128, 2009.

13. Yang H, Yang T, Baur JA, Perez E, Matsui T, Carmona JJ, Lamming DW, Souza-Pinto NC, Bohr VA, Rosenzweig A et al: Nutrient-sensitive mitochondrial NAD ${ }^{+}$levels dictate cell survival. Cell 130: 1095-1107, 2007.

14. Chang HC and Guarente L: SIRT1 mediates central circadian control in the SCN by a mechanism that decays with aging. Cell 153: 1448-1460, 2013.

15. Luo $X$ and Kraus WL: On PAR with PARP: Cellular stress signaling through poly(ADP-ribose) and PARP-1. Genes Dev 26 417-432, 2012.

16. Schreiber V, Dantzer F, Ame JC and de Murcia G: Poly(ADP-ribose): Novel functions for an old molecule. Nat Rev Mol Cell Biol 7: 517-528, 2006.

17. Braidy N, Guillemin GJ, Mansour H, Chan-Ling T, Poljak A and Grant R: Age related changes in $\mathrm{NAD}^{+}$metabolism oxidative stress and Sirt1 activity in wistar rats. PLoS One 6: e19194, 2011

18. Prolla TA and Denu JM: NAD ${ }^{+}$deficiency in age-related mitochondrial dysfunction. Cell Metab 19: 178-180, 2014.
19. Mouchiroud L, Houtkooper RH, Moullan N, Katsyuba E, Ryu D, Cantó C, Mottis A, Jo YS, Viswanathan M, Schoonjans K, et al: The $\mathrm{NAD}(+) /$ sirtuin pathway modulates longevity through activation of mitochondrial UPR and FOXO signaling. Cell 154: 430-441, 2013.

20. Mills KF, Yoshida S, Stein LR, Grozio A, Kubota S, Sasaki Y, Redpath P, Migaud ME, Apte RS, Uchida K, et al: Long-term administration of nicotinamide mononucleotide mitigates age-associated physiological decline in mice. Cell Metab 24: 795-806, 2016

21. Zhang H, Ryu D, Wu Y, Gariani K, Wang X, Luan P, D'Amico D, Ropelle ER, Lutolf MP, Aebersold R, et al: $\mathrm{NAD}^{+}$repletion improves mitochondrial and stem cell function and enhances life span in mice. Science 352: 1436-1443, 2016.

22. Fang EF, Kassahun H, Croteau DL, Scheibye-Knudsen M, Marosi K, Lu H, Shamanna RA, Kalyanasundaram S, Bollineni RC, Wilson MA, et al: $\mathrm{NAD}(+)$ replenishment improves lifespan and healthspan in ataxia telangiectasia models via mitophagy and DNA repair. Cell Metab 24: 566-581, 2016.

23. Camacho-Pereira J, Tarragó MG, Chini CC, Nin V, Escande C, Warner GM, Puranik AS, Schoon RA, Reid JM, Galina A, et al: CD38 dictates age-related NAD decline and mitochondrial dysfunction through an SIRT3-dependent mechanism. Cell Metab 23: 1127-1139, 2016.

24. Mei SC and Brenner C: NAD as a genotype-specific drug target. Chem Biol 20: 1307-1308, 2013.

25. Chiarugi A, Dölle C, Felici R and Ziegler M: The NAD metabolome - a key determinant of cancer cell biology. Nat Rev Cancer 12: 741-752, 2012.

26. Gallí M, Van Gool F, Rongvaux A, Andris F and Leo O: The nicotinamide phosphoribosyltransferase: A molecular link between metabolism, inflammation, and cancer. Cancer Res 70: 8-11, 2010.

27. Garten A, Petzold S, Körner A, Imai S and Kiess W: Nampt: Linking NAD biology, metabolism and cancer. Trends Endocrinol Metab 20: 130-138, 2009.

28. Hasmann M and Schemainda I: FK866, a highly specific noncompetitive inhibitor of nicotinamide phosphoribosyltransferase, represents a novel mechanism for induction of tumor cell apoptosis. Cancer Res 63: 7436-7442, 2003.

29. Khan JA, Tao X and Tong L: Molecular basis for the inhibition of human NMPRTase, a novel target for anticancer agents. Nat Struct Mol Biol 13: 582-588, 2006.

30. Thakur BK, Dittrich T, Chandra P, Becker A, Kuehnau W, Klusmann JH, Reinhardt D and Welte K: Involvement of p53 in the cytotoxic activity of the NAMPT inhibitor FK866 in myeloid leukemic cells. Int J Cancer 132: 766-774, 2013.

31. Thakur BK, Dittrich T, Chandra P,Becker A,Lippka Y, Selvakumar D, Klusmann JH, Reinhardt D and Welte K: Inhibition of NAMPT pathway by FK866 activates the function of p53 in HEK293T cells. Biochem Biophys Res Commun 424: 371-377, 2012.

32. Schuster S, Penke M, Gorski T, Gebhardt R, Weiss TS, Kiess W and Garten A: FK866-induced NAMPT inhibition activates AMPK and downregulates mTOR signaling in hepatocarcinoma cells. Biochem Biophys Res Commun 458: 334-340, 2015.

33. Cea M, Cagnetta A, Fulciniti M, Tai YT, Hideshima T, Chauhan D, Roccaro A, Sacco A, Calimeri T, Cottini F, et al: Targeting $\mathrm{NAD}^{+}$salvage pathway induces autophagy in multiple myeloma cells via mTORC1 and extracellular signal-regulated kinase (ERK1/2) inhibition. Blood 120: 3519-3529, 2012.

34. Billington RA, Genazzani AA, Travelli $C$ and Condorelli F: NAD depletion by FK866 induces autophagy. Autophagy 4: 385-387, 2008.

35. Xu R, Tian E, Tang H, Liu C and Wang Q: Proteomic analysis of gossypol induces necrosis in multiple myeloma cells. Biomed Res Int 2014: 839232, 2014.

36. Brigelius-Flohé R and Maiorino M: Glutathione peroxidases. Biochim Biophys Acta 1830: 3289-3303, 2013.

37. Brigelius-Flohé R and Kipp A: Glutathione peroxidases in different stages of carcinogenesis. Biochim Biophys Acta 1790: $1555-1568,2009$.

38. Novoselov SV, Kryukov GV, Xu XM, Carlson BA, Hatfield DL and Gladyshev VN: Selenoprotein $\mathrm{H}$ is a nucleolar thioredoxin-like protein with a unique expression pattern. J Biol Chem 282: 11960-11968, 2007.

39. Zhang T, Berrocal JG, Yao J, DuMond ME, Krishnakumar R, Ruhl DD, Ryu KW, Gamble MJ and Kraus WL: Regulation of poly(ADP-ribose) polymerase-1-dependent gene expression through promoter-directed recruitment of a nuclear $\mathrm{NAD}^{+}$ synthase. J Biol Chem 287: 12405-12416, 2012.

40. Bai P, Cantó C, Oudart H, Brunyánszki A, Cen Y, Thomas C, Yamamoto H, Huber A, Kiss B, Houtkooper RH, et al: PARP-1 inhibition increases mitochondrial metabolism through SIRT1 activation. Cell Metab 13: 461-468, 2011. 